\title{
Performance of Monte Carlo Event Generators for the Production of Boson and Multi-Boson States ATLAS Analysis
}

\author{
Francesco Giuli*广 \\ University of Oxford \\ E-mail: francesco.giuli@cern.ch, francesco.giuli@physics.ox.ac.uk
}

\begin{abstract}
The Monte Carlo (MC) setups used by ATLAS to model boson+jets and multi-boson processes at $\sqrt{s}=13 \mathrm{TeV}$ in proton-proton collisions are described. Comparisons between data and several event generators are provided for key kinematic distributions. Issues associated with the evaluation of systematic uncertainties are also discussed. This proceeding is a summary of the results collected in two recent ATLAS PUB notes published for the MC workshop jointly-organised by the the ATLAS and CMS Collaborations and held at CERN in May 2017.
\end{abstract}

EPS-HEP 2017, European Physical Society conference on High Energy Physics 5-12 July 2017

Venice, Italy

* Speaker.

${ }^{\dagger}$ on behalf of the ATLAS Collaboration 
Introduction This proceeding describes the Monte Carlo (MC) used by ATLAS [1] to model boson + jets $(V+$ jets $)[2]$ and multi-boson $(V V / V V V)$ [3] processes in $13 \mathrm{TeV} p p$ collisions. The baseline MC generators are compared with each other in key kinematic distributions of the processes under study. Sample normalisation and assignment of systematic uncertainties are discussed.

Boson+jets The setup of the samples used for the different comparisons is described in the following:

- Sherpa Sherpa [4] is a parton shower MC generator simulating additional hard parton emissions that are matched to a parton shower based on Catani-Seymour subtraction terms [5]. The merging of multi-parton matrix elements (ME) with the parton shower (PS) is achieved using an improved CKKW matching procedure [6, 7], which is extended to next-to-leading order (NLO) accuracy using the MEPS@NLO prescription [8].

- MadGraph5_aMC@NLO using CKKW-L Matrix elements for $V+$ up to 4 partons at LO accuracy are produced using MadGraph5_aMC@NLO v2.2.2 [9] interfaced with Pythia v8.186 [10] for the modelling of the parton shower and underlying event. The CKKW-L matching $[6,11]$ and merging procedure is applied, with a merging scale $\left(\mu_{Q}\right)$ of 30 GeV. The NNPDF3.0NLO PDF set is used with $\alpha_{s}=0.118$ and the A14 tune of Pythia8 [12] is applied.

- MadGraph5_aMC@NLO using FxFx Samples have also been generated using the MadGraph5_aMC@NLO program to generate matrix elements for $V+0,1$ and 2 partons to NLO accuracy. The showering and subsequent hadronisation has been performed using Pythia 8.210 with the A14 tune, using the NNPDF2.3LO PDF set with $\alpha_{s}=0.13$. The different jet multiplicities are merged using the FxFx prescription [13] implemented in the MadGraph5_aMC@NLO program (version 2.3.3 is used here). The impact of various $\mu_{Q}$ has been studied, analysing three different values: $20 \mathrm{GeV}$ (downward variation), $25 \mathrm{GeV}$ (nominal value) and $50 \mathrm{GeV}$ (upward variation).

- Powheg MINLO Predictions from Powheg MinLo $[14,15,16]$ interfaced to Pythia 8.210 [10] with the AZNLO tune [17] were obtained to produce $V+$ jets events. The PDF set used in Powheg is CT14NNLO [18] whereas the PDF set used in the parton shower is the CTEQ6L1 [19] LO set.

Figure 1 shows comparisons of Sherpa 2.2 (red), Powheg MiNLO+Py8 (blue) and MG5_aMC@NLO+Py 8 using FxFx (green) against data (black) from the $13 \mathrm{TeV}$ ATLAS $Z+$ jets measurement [20]. The measurement uncertainty is indicated by a grey band in the ratio panels, while generator uncertainties have been estimated using Sherpa 2.2 and are indicated by the orange band in the ratio panels. The MEPS@NLO setup using Sherpa 2.2 is NLO-accurate for up to two extra emissions and LO-accurate for up to four extra emissions. At very high jet multiplicities, Sherpa 2.2 starts to diverge from the data, indicating too much activity in the PS. The MG5_aMC@NLO setup already begins to mismodel the data for lower multiplicities, as it lacks additional multilegs beyond the third emission, making it less attractive for many new-physics searches interested in a good modelling of high multijet configurations, even though the mismodelling is likely covered by 


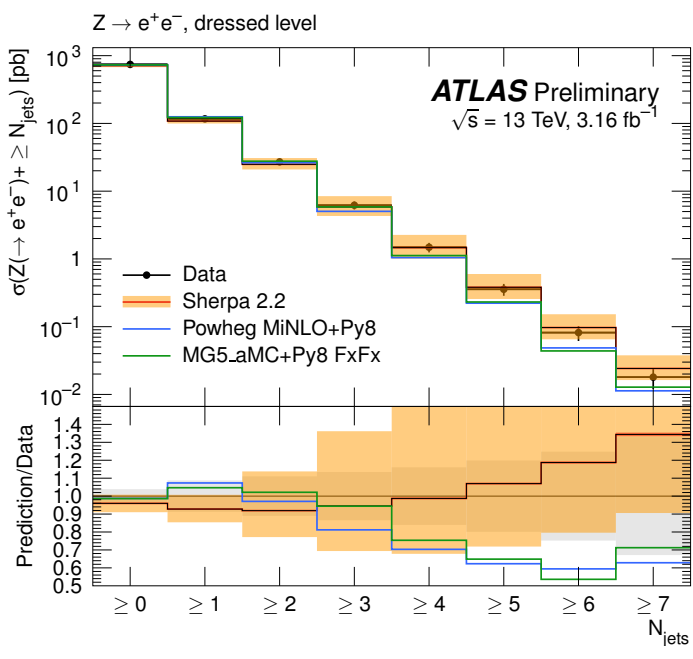

(a)

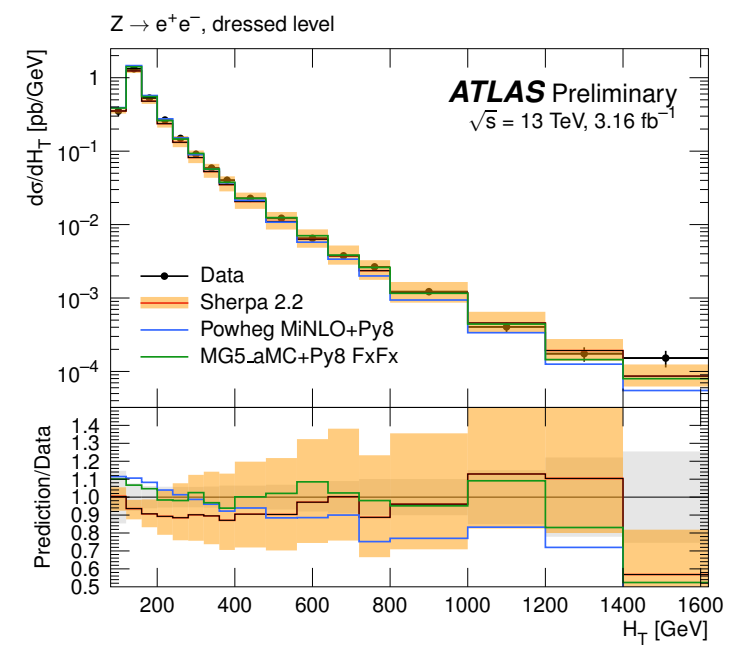

(b)

Figure 1: Predictions for the differential cross sections as a function of the inclusive jet multiplicity (left) and of the scalar jet- $p_{\mathrm{T}}$ sum, $H_{\mathrm{T}}$, (right) from Sherpa 2.2 (red), Powheg MiNLO+Pythia8 (blue) and MG5_aMC@NLO+Py 8 using FxFx (green). The predictions are compared to data (black) from a recent ATLAS measurement of $Z+$ jets production [20]. These plots are taken from Ref. [2].

the scale uncertainties. A similar comparison for the first- and third-order splitting scale distribution occuring in the $k_{t}$ algorithm [21,22] is shown in Figure 2 between Sherpa 2.2 (red) and MG5_aMC@NLO+Py 8 using FxFx (blue) at a centre-of-mass energy of $13 \mathrm{TeV}$. Generator uncertainties have been estimated using Sherpa 2.2 and are indicated by the orange band in the ratio panels. In addition, variations of the FxFx matching scale, $\mu_{Q}$, (shown in solid and dashed green) have been studied for the MG5_aMC@NLO+Py 8 setup. The statistical uncertainty component is indicated by the error bars. It can be seen that neither the PDF and scale variations estimated using Sherpa 2.2 nor the matching-scale variations estimated for the FxFx setup can cover the differences between the two predictions seen in the transition region, e.g. $\sqrt{d_{k}} \approx 5 \mathrm{GeV}$, between the soft and the perturbative regimes, where $\sqrt{d_{k}}$ is the associated splitting scale defined in Ref. [23].

\section{Multi-boson}

Fully leptonic diboson processes The processes can be grouped according to the number of charged leptons, giving rise to the following final states: $4 \ell, 3 \ell v, 2 \ell 2 v, \ell 3 v$ and $4 v$. An overview of the accuracy achieved with the chosen generators is given in Table 1. A general shape comparison between PowhegBox and Sherpa v2.2 is shown in Figure 3(a).

The distributions are normalised to the samples' cross section and there is no reweighting applied.

\begin{tabular}{cccccc}
\hline & $V V+0 j$ & $V V+1 j$ & $V V+2 j$ & $V V+3 j$ & $V V+\geq 4 j$ \\
\hline Sherpa v2.2 & NLO & NLO & LO & LO & PS \\
PowhegBox+PYTHIA8/Herwig++ & NLO & LO & PS & PS & PS \\
MadGraph5_aMCenLO+PYTHIA8 & NLO & NLO & LO & PS & PS \\
\hline
\end{tabular}

Table 1: Overview of process accuracies for the chosen generators. 


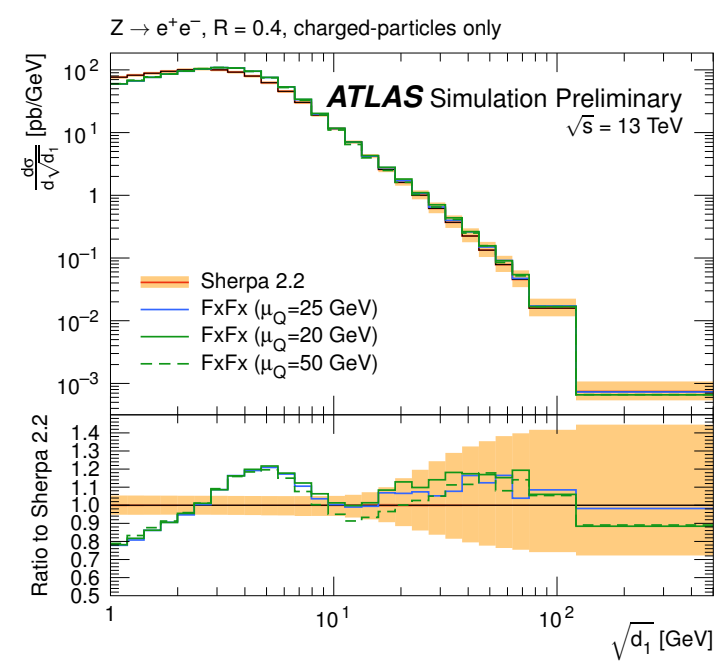

(a)

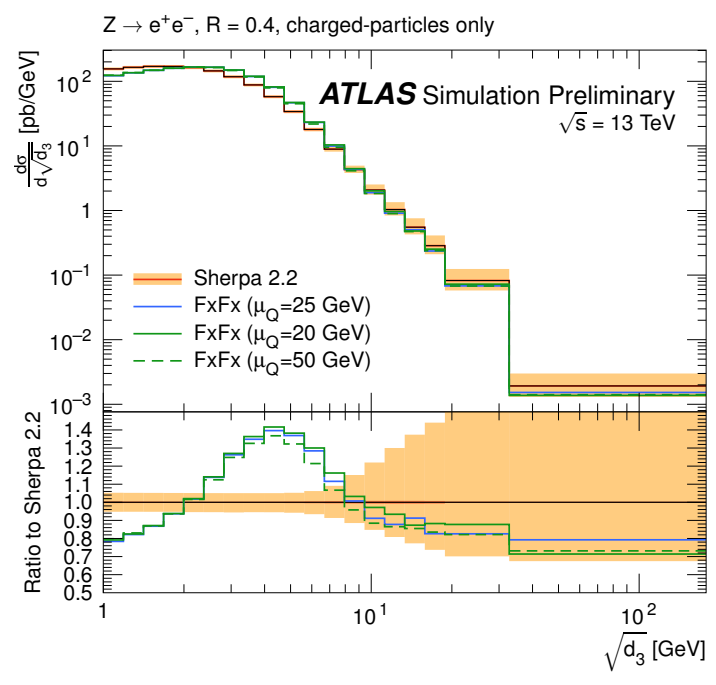

(b)

Figure 2: Predictions for the differential cross sections as a function of first-order splitting scale, $\sqrt{d_{1}}$ (left), and of the third-order splitting scale, $\sqrt{d_{3}}$ (right), in the $k_{t}$ algorithm using a jet-radius parameter of $R=$ 0.4 [23] from Sherpa 2.2 (red) and MG5_amcenLO using FxFx using $\mu_{Q}$ of $20 \mathrm{GeV}$ (blue), $25 \mathrm{GeV}$ (solid green) and $50 \mathrm{GeV}$ (dashed green). These plots are taken from Ref. [2].

In Figure 3(a), the four-lepton invariant mass, which is an observable quite insensitive to higherorder QCD effects, shows good agreement above the ZZ threshold between the two generators. The deviations below this threshold can be related to differences in both the QCD and the electroweak shower which is fully based on Pythia 8 in case of PowhegBox while Sherpa uses its own shower model. Higher-order electroweak corrections have been studied and it has been found that they can also significantly affect several observables, particularly in the tails of the distributions. In Figure 3(b), a comparison of the differential cross section $\left(d \sigma / d p_{\mathrm{T}}^{\mathrm{ZZ}}\right)$ predicted by Sherpa and PowhegBox is shown. While the predictions of both generators are similarly corrected for higherorder electroweak effects, there is an additional reweighting applied for PowhegBox intended to bring it from NLO to approximately NNLO QCD accuracy.

Semi leptonic diboson processes As regards the generators setup, an overview is given in Table 2. Figure 4 focuses on the comparison among different generators for the invariant mass of the reconstructed hadronically decaying boson, $m\left(j_{1}, j_{2}\right)$, for all diboson processes considered. It

\begin{tabular}{lcccccc}
\hline & & $V V+0 j$ & $+1 j$ & $+2 j$ & $+3 j$ & $+\geq 4 j$ \\
\hline \multirow{4}{*}{$V V=W W, W Z$} & Sherpa v2.1.1 & NLO & LO & LO & LO & PS \\
& Sherpa v2.2 & NLO & NLO & LO & LO & PS \\
& PowhegBox+Py8/Herwig++ & NLO & LO & PS & PS & PS \\
\hline \multirow{3}{*}{$V V=Z Z$} & Sherpa v2.1.1 & NLO & NLO & LO & LO & PS \\
& Sherpa v2.2 & NLO & NLO & LO & LO & PS \\
& PowhegBox+Py8/Herwig++ & NLO & LO & PS & PS & PS \\
\hline
\end{tabular}

Table 2: Overview of process accuracies for the chosen generators.

has been found that the PowhegBox predictions are consistent with each other, indipendent of the 


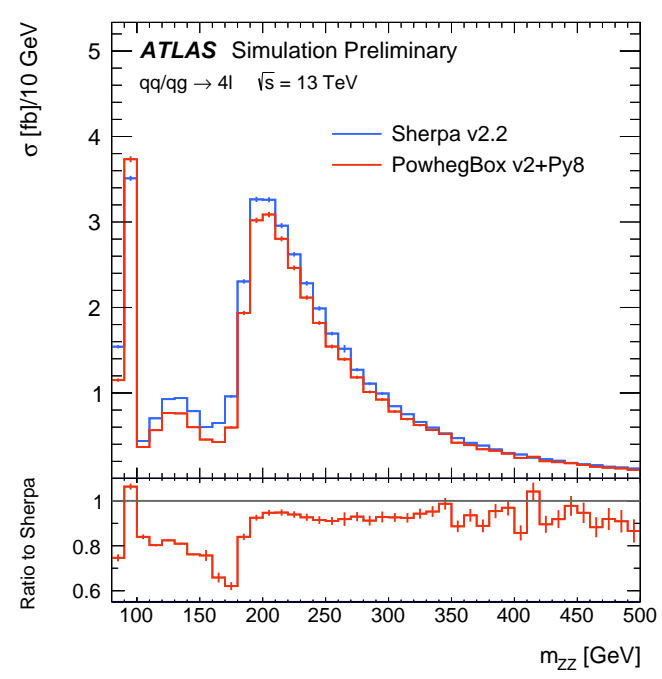

(a)

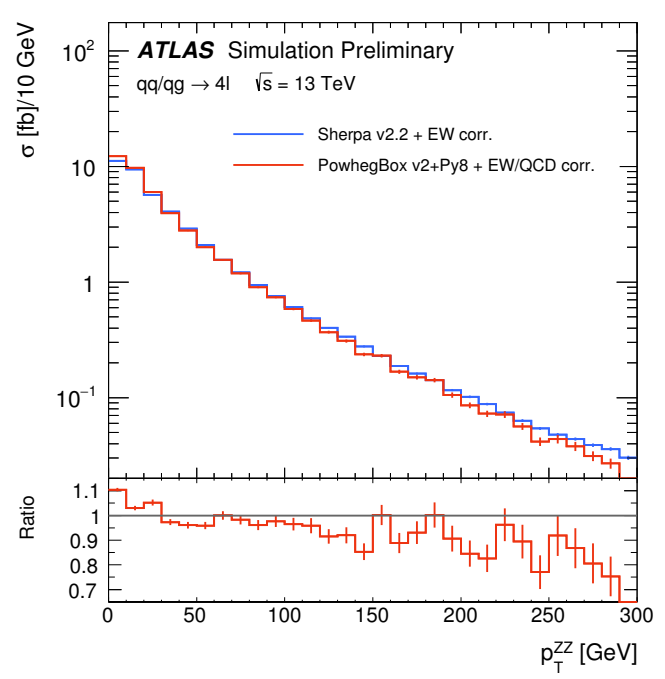

(b)

Figure 3: (a) Comparison of shapes $\left(d \sigma / d m_{Z Z}\right)$ normalised to the samples' cross section and combining all four channels as predicted by PowhegBox and Sherpa; (b) Differential cross section predictions $\left(d \sigma / d p_{T T}\right)$ of Sherpa and PowhegBox both corrected for higher-order electroweak effects and additional incorporation of QCD effects in PowhegBox. These plots are taken from Ref. [3].

choice from the program used to implement the showering and that they are, in general, softer than the Sherpa v2.2 ones. A similar conclusion can be drawn if analysing the $W Z(\rightarrow \ell \ell)$ and the $W Z(\rightarrow v v)$ channels.

Electroweak diboson production with jets Electroweak diboson production with at least two jets includes vector boson scattering (VBS) diagrams, where the two "tagging" jets recoil against the (heavy) gauge bosons. The resulting leptonic final states include the $4 \ell j j$, as well as the $2 \ell 2 v j j$ final states, where the two lepton charges can be the same or opposite sign. An overview of the accuracy achieved with the chosen generators is given in Table 3. A comparison of pre-

Table 3: Accuracies of the chosen generators for the listed electroweak processes.

\begin{tabular}{lcccc}
\hline & & $V V+2 j$ & $V V+3 j$ & $V V+\geq 4 j$ \\
\hline \multirow{2}{*}{$V V j j=\ell^{ \pm} \ell^{\mp} 2 v j j$} & VBFNLO+Py8 & LO & PS & PS \\
& MG5_aMC@NLO+Py8 & LO & PS & PS \\
\hline \multirow{2}{*}{$V V j j=\ell^{ \pm} \ell^{ \pm} 2 v j j$} & Sherpa v2.1.1 & LO & PS & PS \\
& PowhegBox+Py8 & NLO & LO & PS \\
\hline
\end{tabular}

dicted kinematic distributions from MG5_aMC@NLO and VBFNLO, both showered with Pythia8 is shown in Figure 5: these distributions are found to be similar.

Furthermore, the comparison between the leading jet $p_{\mathrm{T}}$ distribution predicted by PowhegBox and Sherpa can be found in Figure 6(a); PowhegBox prediction is overall in agreement within uncertainties with what is seen in Sherpa. The systematic variations for events generated with PowhegBox are derived using the PowhegBox internal reweighting scheme. The resulting 


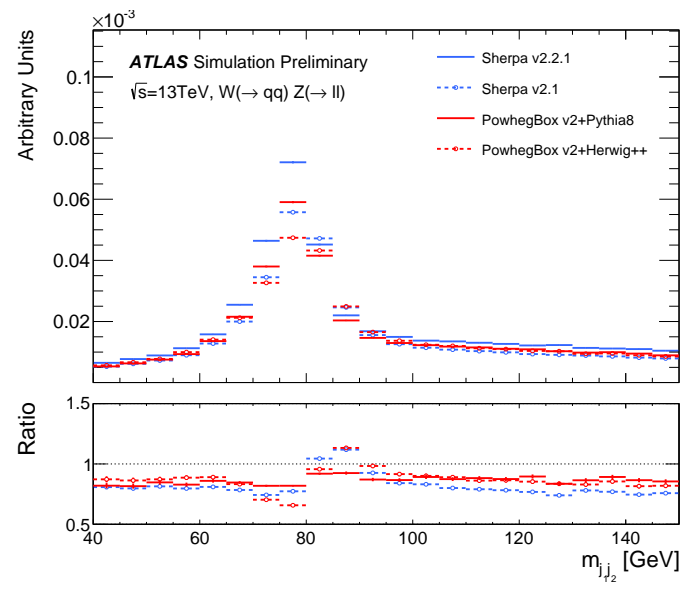

(a)

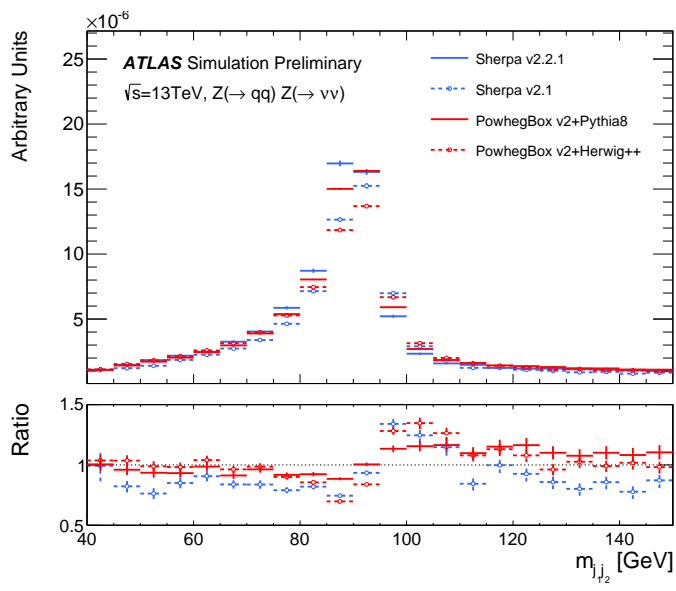

(b)

Figure 4: Comparison of the invariant dijet mass $m\left(j_{1}, j_{2}\right)$ distribution in two different semileptonic final states at $13 \mathrm{TeV}$. The lower panel shows the ratio of each distribution with respect to the Sherpa v2.2 prediction. These plots are taken from Ref. [3].

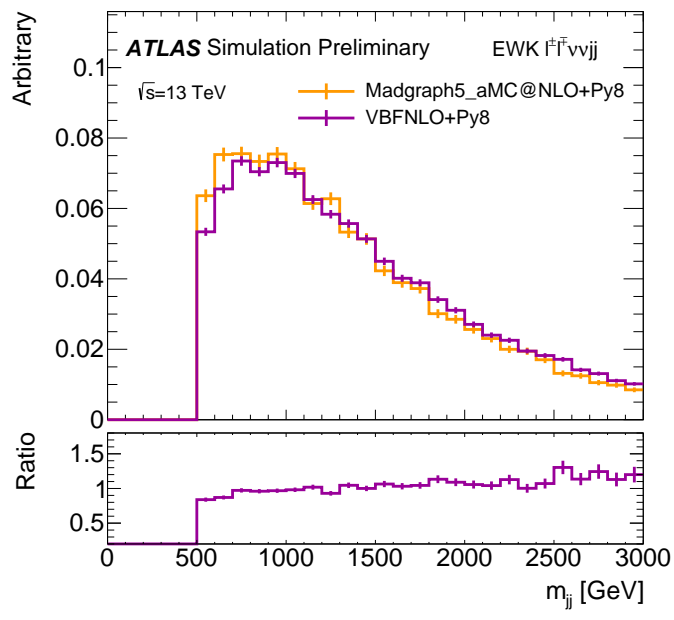

(a)

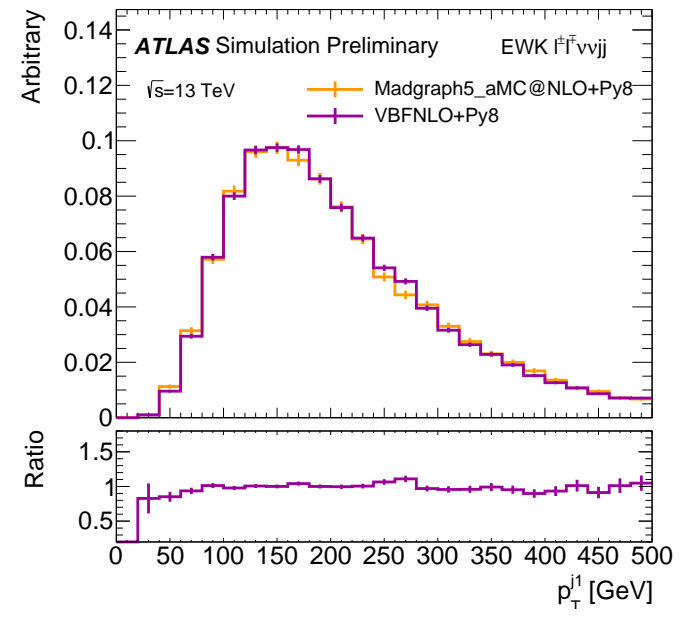

(b)

Figure 5: Comparison of predicted kinematic distributions from MG5_aMC@NLO and VBFNLO, both showered with Pythia 8, for $m_{j j}$ (left) and leading jet $p_{\mathrm{T}}$ (right). These plots are taken from Ref. [3].

weights include renormalisation scale and factorisation scale. There are eight scale variations corresponding to factors of $1 / 2$ or 2 applied independently of the renormalisation and factorisation scales, as shown in Figure 6(b) for the dijet system invariant mass distribution. The impact of scale variation is moderate (2-3\%) for $m_{j_{1}, j_{2}}$ up to $4 \mathrm{TeV}$, and then it becomes larger, rising up to $10-15 \%$ for $m_{j_{1}, j_{2}} \approx 7 \mathrm{TeV}$. 


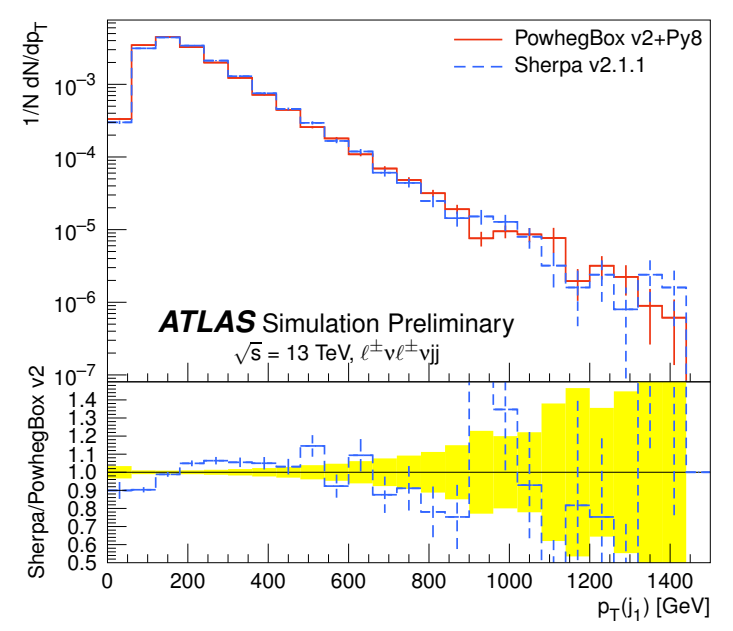

(a)

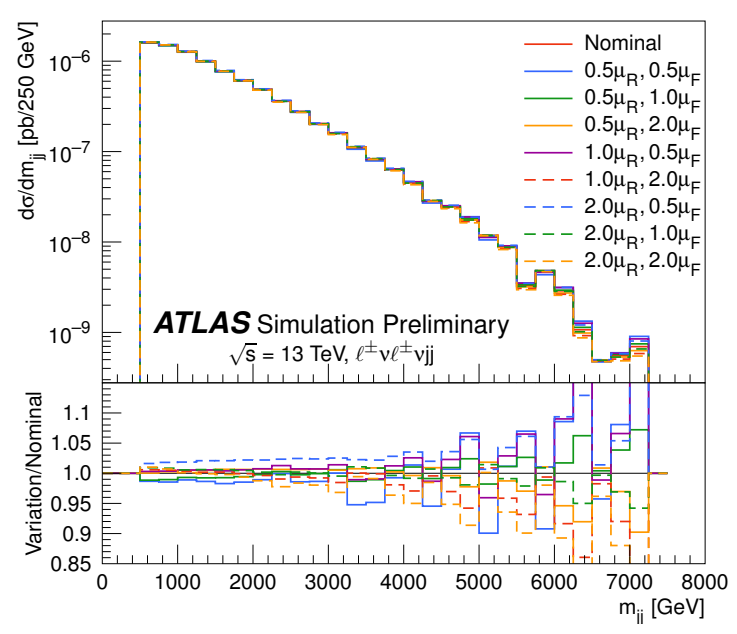

(b)

Figure 6: (a) Comparison between the leading jet $p_{\mathrm{T}}$ distribution predicted by PowhegBox and Sherpa. The yellow band represents the statistical uncertainty of the PowhegBox sample; (b) Impact of scale variations on the dijet invariant mass $m_{j j}$. These plots are taken from Ref. [3].

\section{References}

[1] ATLAS Collaboration, The ATLAS Experiment at the CERN Large Hadron Collider, JINST 3 (2008) S08003, DOI:10.1088/1748-0221/3/08/S08003.

[2] ATLAS Collaboration, ATLAS simulation of boson plus jets processes in Run 2, ATL-PHYS-PUB-2017-006, 2017, URL: https: //cds.cern.ch/record/2261937/.

[3] ATLAS Collaboration, Multi-Boson Simulation for 13 TeV ATLAS Analyses, ATL-PHYS-PUB-2017-005, 2017, URL: https://cds.cern.ch/record/2119986/.

[4] T. Gleisberg et al., Event generation with SHERPA 1.1, JHEP 02 (2009) 007, arXiv:0811.4622 [hep-ph], DOI:10.1088/1126-6708/2009/02/007.

[5] S. Schumann and F. Krauss, A Parton shower algorithm based on Catani-Seymour dipole factorisation, JHEP 03 (2008) 038, arXiv:0709.1027 [hep-ph], DOI:10.1088/1126-6708/2008/03/038.

[6] S. Höche et al., QCD matrix elements and truncated showers, JHEP 05 (2009) 053, arXiv:0903.1219 [hep-ph], DOI:10.1088/1126-6708/2009/05/053.

[7] S. Catani et al., QCD matrix elements + parton showers, JHEP 11 (2001) 063, arXiv:hep-ph/0109231, DOI:10.1088/1126-6708/2001/11/063.

[8] S. Höche et al., QCD matrix elements + parton showers: The NLO case, JHEP 04 (2013) 027, arXiv:1207.5030 [hep-ph], DOI:10.1007/JHEP04(2013)027.

[9] J. Alwall et al., The automated computation of tree-level and next-to-leading order differential cross sections, and their matching to parton shower simulations, JHEP 07 (2014) 079, arXiv:1405.0301 [hep-ph], DOI:10.1007/JHEP07(2014)079.

[10] T. Sjostrand, S. Mrenna and P. Skands, A brief introduction to PYTHIA 8.1, Comput. Phys. Commun. 178 (2008) 852, arXiv:0710.3820 [hep-ph], DOI:10.1016/j.cpc.2008.01.036. 
[11] L. Lonnblad, Correcting the color dipole cascade model with fixed order matrix elements, JHEP 05 (2002) 046, arXiv:hep-ph/0112284, DOI:10.1088/1126-6708/2002/05/046.

[12] ATLAS Collaboration, ATLAS Run 1 Pythia8 tunes, ATL-PHYS-PUB-2014-021, 2014, URL: https://cds.cern.ch/record/1966419.

[13] R. Frederix and S. Frixione, Merging meets matching in MC@NLO, JHEP 12 (2012) 061, arXiv:1209.6215 [hep-ph], DOI:10.1007/JHEP12(2012)061.

[14] S. Alioli et al., A general framework for implementing NLO calculations in shower Monte Carlo programs: the POWHEG BOX, JHEP 06 (2010) 043, arXiv:1002.2581 [hep-ph], DOI:10.1007/JHEP06(2010)043.

[15] K. Hamilton, P. Nason and G. Zanderighi, MINLO: Multi-Scale Improved NLO, JHEP 10 (2012) 155, arXiv:1206.3572 [hep-ph], DOI:10.1007/JHEP10(2012)155.

[16] K. Hamilton et al., Merging $H / W / Z+O$ and 1 jet at $N L O$ with no merging scale: a path to parton shower + NNLO matching, JHEP 05 (2013) 082, arXiv:1212.4504 [hep-ph], DOI:10.1007/JHEP05(2013)082.

[17] ATLAS Collaboration, Example ATLAS tunes of Pythia8, Pythia6 and Powheg to an observable sensitive to Z boson transverse momentum, ATL-PHYS-PUB-2013-017, 2013, URL: https://cds.cern.ch/record/1629317.

[18] S. Dulat et al., New parton distribution functions from a global analysis of quantum chromodynamics, Phys. Rev D93 (2016) 033006, arXiv:1506.07443 [hep-ph], DOI:10.1103/PhysRevD.93.033006.

[19] J. Pumplin et al., New generation of parton distributions with uncertainties from global QCD analysis, JHEP 07 (2002) 012, arXiv:0201195 [hep-ph], DOI:10.1088/1126-6708/2002/07/012.

[20] ATLAS Collaboration, Measurements of the production cross section of a $Z$ boson in association with jets in pp collisions at $\sqrt{s}=13 \mathrm{TeV}$ with the ATLAS detector, Eur. Phys. J. C77 (2017) 361, arXiv: 1702.05725 [hep-ex].

[21] S. Catani et al., Longitudinally invariant $k_{t}$ clustering algorithms for hadron hadron collisions, Nucl. Phys. B406 (1993) 187-224, DOI:10.1016/0550-3213(93)90166-M.

[22] Gavin P. Salam, Towards Jetography, Eur. Phys. J. C67 (2010) 637-686, arXiv:0906.1833 [hep-ph], DOI:10.1140/epjc/s10052-010-1314-6.

[23] ATLAS Collaboration, Measurement of the $k_{\mathrm{t}}$ splitting scales in $Z \rightarrow \ell \ell$ events in pp collisions at $\sqrt{s}=8 \mathrm{TeV}$ with the ATLAS detector, JHEP 08 (2017) 026, arXiv:1704.01530 [hep-ex], DOI:10.1007/JHEP08(2017)026. 